\title{
Complexes of Domain Walls in One-Dimensional Ferromagnets Near and Far from Phase Transition
}

\author{
A. JANUTKA* \\ Institute of Physics, Wrocław University of Technology, Wybrzeże Wyspiańskiego 27, 50-370 Wrocław, Poland \\ (Received November 30, 2012; in final form March 27, 2013) \\ Interactions of domain walls are analyzed with relevance to formation of stationary bubbles (bound state of \\ two domain walls) and bound states of many domains in one-dimensional systems. We investigate the domain \\ structures in ferromagnets which are described with the Landau-Lifshitz equation as well as the domains in critical \\ systems described with the Ginzburg-Landau equation. Supplementing previous author studies on the creation of \\ hard bubbles [formed by one Bloch domain wall and one Néel (Ising) domain wall] in the presence of an external \\ (magnetic) field, the soft bubbles consisting of two Bloch domain walls or two Néel (Ising) domain walls are studied \\ in detail. The interactions of two domain walls of the same kind are studied in the framework of a perturbation \\ calculus.
}

DOI: $10.12693 /$ APhysPolA.124.23

PACS: 05.45.Yv, 64.60.Ht, 75.70.Kw, 75.78.Fg, 77.80.Dj

\section{Introduction}

Localized and patterned (labyrinth) structures in bistable media are widely considered with relevance to the storage of binary information $[1,2]$. Far enough from the phase-transition point, the complexes of domain walls (DWs) are observed in chemical reactors, magnetic and polar (solid and liquid) media while their basic properties are described with equations of motion with are similar for different media. In particular, far from the critical regime, the magnetization (polarization) dynamics is described with the Landau-Lifshitz-Gilbert (LLG) equation while near the criticality it is governed by the Ginzburg-Landau (GL) equation. Recently, one observes an especial interest in 1D states of many magnetic and polar DWs due to hopes for miniaturization of memory carriers which result from achievements of current nanowire technology [3-5]. It gave a new pulse for continuing research on the methods of controlling the motion of DWs in ferromagnetic wires whose intensive studies have begun over four decades ago motivated by the technology of magnetic bubbles in 2D systems [6], and currently induces the interest in the driven motion of DWs in ferroelectric nanostructures [7]. The problem of stability of many-domain states is connected to the need of switching the domain-encoded binary information on demand since such process induces unbalanced interactions of the DWs.

In the present work, I analyze binary interactions between the DWs (of the Bloch and Néel-Ising type) with dependence on the distance of their separation and their chiralities (opposite or like). It enables me to study the formation of magnetization bubbles in 1D (stationary states of two DWs are 1D counterparts of the magne-

*e-mail: Andrzej.Janutka@pwr.wroc.pl tization bubbles of planar ferromagnets) far from and near the criticality and to consider the stability of many-domain structures. With correspondence to $2 \mathrm{D}$ magnetic bubbles (which are called hard ones when their boundary is composed of alternating Bloch and Néel lines), I divide the 1D bubbles into hard ones (composed of one Bloch and one Néel-Ising DWs) and soft ones (composed of two Bloch DWs or two Néel-Ising DWs) $[8,9]$. In my recent papers on the externally-driven motion of DWs, I have studied the formation of 1D hard bubbles via collision of a Bloch DW with a Néel (Ising) DW in the normal and critical regimes (solving the LLG and GL equations, respectively) $[10,11]$. Here, the energy of soft bubbles is studied as function of the bubble length within a perturbation calculus in order to complete the picture of the DW interactions. I follow a perturbation approach to the DW interaction developed in Ref. [12] with relevance to the parametrically-driven nonlinear Schrödinger equation. It differs from previous ones (e.g. [13]) in terms of the perturbation expansion of the dynamical parameter (magnetization) whose present form ensures conservation of the magnetization length.

With application to ferromagnets, the 1D approximation is relevant to crystalline nanowires with strong bulk anisotropy compared to surface magnetostatic effects (and, especially, with circular cross-section). The present analysis provides a basis for comparison of the DW interactions in such magnets to the interactions of DWs in the nanostripes of soft ferromagnets which are studied in Ref. [14].

Binary interactions of the DWs and the stability of 1D bubbles are considered in Sects. 2 and 3, with relevance to the ferromagnetic wire at low temperatures and to the subcritical systems, respectively. In Sect. 4 , the stability of many-DW 1D systems is considered on the basis of previous-section results. 


\section{Bubbles of magnetic DWs at low temperatures}

I consider DW solutions to the LLG equation applied to the exchange ferromagnet with the two-axis anisotropy

$$
\begin{gathered}
\frac{\partial \boldsymbol{m}}{\partial t}=\frac{J}{M} \boldsymbol{m} \times \frac{\partial^{2} \boldsymbol{m}}{\partial x^{2}}+\gamma \boldsymbol{m} \times \boldsymbol{H}+\frac{\beta_{1}}{M}(\boldsymbol{m} \cdot \hat{i}) \boldsymbol{m} \times \hat{i} \\
-\frac{\beta_{2}}{M}(\boldsymbol{m} \cdot \hat{j}) \boldsymbol{m} \times \hat{j}-\frac{\alpha}{M} \boldsymbol{m} \times \frac{\partial \boldsymbol{m}}{\partial t},
\end{gathered}
$$

which is a completed (via including the external magnetic field and damping) system that was originally studied in [15]. The first term of the right hand side (rhs) of (1) relates to the exchange spin interaction while the second (Zeeman) term depends on the external magnetic field $\boldsymbol{H}=\left(H_{x}, 0,0\right)$, thus, $\gamma$ denotes the gyromagnetic factor while $J$ represents the exchange stiffness constant (usually denoted by $A$ ) up to the renormalization; $J=2 \gamma A / M$. The constant $\beta_{1(2)}$ determines strength of the easy axis (plane) anisotropy and $\hat{i} \equiv(1,0,0)$, $\hat{j} \equiv(0,1,0)$. I notice that the long axis of a nanowire is an easy axis for the most often investigated magnets, however, another choice of the anisotropy axes does not influence the magnetization dynamics (the model can be applied to the so-called nanowires with perpendicular magnetic anisotropy [16]). The Gilbert damping constant is denoted by $\alpha$. Since LLG equation is valid only when the constraint $|\boldsymbol{m}|=M$ is satisfied, one writes equations of the unconstrained dynamics equivalent to (1). Introducing $m_{ \pm} \equiv m_{y} \pm \mathrm{i} m_{z}$, one represents the magnetization components using a pair of complex functions $g(x, t), f(x, t)$. The relation between the primary and secondary dynamical variables

$$
m_{+}=\frac{2 M}{f^{*} / g+g^{*} / f}, \quad m_{x}=M \frac{f^{*} / g-g^{*} / f}{f^{*} / g+g^{*} / f}
$$

ensures that $|\boldsymbol{m}|=M$. Inserting (2) into (1) leads, following the Hirota method for solving nonlinear equations $[15,17]$, to

$$
\begin{aligned}
f[ & \left.-\mathrm{i} D_{t}+J D_{x}^{2}+\alpha D_{t}\right] f^{*} \cdot g+J g^{*} D_{x}^{2} g \cdot g \\
& -\left(\gamma H_{x}+\beta_{1}+\frac{\beta_{2}}{2}\right)|f|^{2} g-\frac{\beta_{2}}{2} f^{* 2} g^{*}=0, \\
g^{*} & {\left[-\mathrm{i} D_{t}-J D_{x}^{2}+\alpha D_{t}\right] f^{*} \cdot g-J f D_{x}^{2} f^{*} \cdot f^{*} } \\
& +\left(-\gamma H_{x}+\beta_{1}+\frac{\beta_{2}}{2}\right)|g|^{2} f^{*}+\frac{\beta_{2}}{2} g^{2} f=0,
\end{aligned}
$$

where $D_{t}, D_{x}$ denote the Hirota operators of differentiation which are defined by

$$
\begin{aligned}
& D_{t}^{m} D_{x}^{n} b(x, t) \cdot c(x, t) \equiv\left(\partial / \partial t-\partial / \partial t^{\prime}\right)^{m} \\
& \quad \times\left.\left(\partial / \partial x-\partial / \partial x^{\prime}\right)^{n} b(x, t) c\left(x^{\prime}, t^{\prime}\right)\right|_{x=x^{\prime}, t=t^{\prime}}
\end{aligned}
$$

The above Hirota reduction of the LLG equation is indicated in order to provide an easy way of verification of below used DW solutions to the original equation.

For $\boldsymbol{H}=0$, the stationary single-DW solutions to (3) take the form

$$
f=1, \quad g=w \mathrm{e}^{k x}, \quad k=k^{*},
$$

where $|k|=\sqrt{\beta_{1} / J}, w=-w^{*}($ a Bloch DW) or $|k|=$ $\sqrt{\left(\beta_{1}+\beta_{2}\right) / J}, w=w^{*}$ (a Néel DW). Let us define $\varphi$ following $\mathrm{e}^{\mathrm{i} \varphi}=w /|w|$, thus, $\varphi= \pm \pi / 2$ for Bloch DW while $\varphi=0, \pi$ for the Néel DW. Note that another nomenclature is often used, one calls the Bloch DWs as the Néel DWs and vice versa (I follow [17]).

Since neither exact double-Bloch nor double-Néel solutions to the LLG equation are not known for the case of zero external field (the length of the soft bubbles diverges with $H_{x} \rightarrow 0$ [18-20]), I analyze the interactions of the relevant DW pairs (the pair of the Bloch DWs and the pair of the Neel DWs) within a perturbation calculus. Locally, in the vicinity of the center of $j$-th DW $(j=1,2)$, one can write the magnetization in the form

$$
\boldsymbol{m}(x, 0)=\boldsymbol{m}^{(j)}(x)+\delta \boldsymbol{m}^{(j)}(x),
$$

where $\boldsymbol{m}^{(j)}$ denotes a stationary single-DW solution to (1) [which corresponds to (4)]

$$
\begin{aligned}
& m_{+}^{(j)}(x)=M \mathrm{e}^{\mathrm{i} \varphi_{j}} \operatorname{sech}\left(\sigma_{j} k\left(x-x_{0 j}\right)\right), \\
& m_{x}^{(j)}(x)=-M \tanh \left(\sigma_{j} k\left(x-x_{0 j}\right)\right),
\end{aligned}
$$

while $\delta \boldsymbol{m}^{(j)}$ denotes a perturbation due to the presence of another DW. Here $\sigma_{1}=-\sigma_{2}= \pm 1, \varphi_{1}= \pm \varphi_{2}$ and $\left|\varphi_{j}\right|=\pi / 2$ (a pair of the Bloch DWs) or $\varphi_{j}=0, \pi$ (a pair of the Néel DWs). When I assume $k>0, \sigma_{j}=1$ relates to the head-to-head spin structure while $\sigma_{j}=-1$ to the tail-to-tail structure. I apply the perturbation of the form

$$
\begin{gathered}
\delta \boldsymbol{m}^{(j)}=\left( \pm \frac{m_{x}^{(k)}}{M}-1\right) \boldsymbol{m}^{(j)} \pm \frac{m_{x}^{(j)}}{M}\left(0, m_{y}^{(k)}, m_{z}^{(k)}\right) \\
\mp \frac{1}{M}\left(m_{y}^{(k)} m_{y}^{(j)}+m_{z}^{(j)} m_{z}^{(k)}, 0,0\right),
\end{gathered}
$$

where $k \neq j$, while plus and minus relate to the bubble magnetization parallel and antiparallel to $x$-axis, respectively. It is motivated by necessity of satisfying the constraint $|\boldsymbol{m}|=M$. The ansatz (7) leads to

$$
\begin{aligned}
& m_{x}= \pm \frac{1}{M}\left[m_{x}^{(1)} m_{x}^{(2)}-\frac{1}{2}\left(m_{+}^{(1)} m_{-}^{(2)}+m_{+}^{(2)} m_{-}^{(1)}\right)\right], \\
& m_{+}= \pm \frac{1}{M}\left(m_{+}^{(1)} m_{x}^{(2)}+m_{+}^{(2)} m_{x}^{(1)}\right)
\end{aligned}
$$

and it is easy to check that the above constraint is fulfilled by any pair of the Bloch DWs or a pair of the Néel DWs since then the product $m_{+}^{(1)} m_{-}^{(2)}$ takes real values (while (8) would cause $|\boldsymbol{m}| \neq M$ in the case of interacting the Bloch DW with the Néel DW). The interaction of topological solitons (DWs) is not simply due to their overlap while it is accompanied by some reorientation of the whole separating them domain (unlike the interaction of pulses). With the condition $|\boldsymbol{m}|=M$, this domain reorientation is included in a realistic way. In a result of its application, the domain reorientation is reflected by the fact that the magnetization (8) contains only products of the components of $\boldsymbol{m}^{(1)}$ and $\boldsymbol{m}^{(2)}$ while it does not contain linear in $\boldsymbol{m}^{(j)}$ terms. The perturbation (7) satisfies the requirement of being small when the interacting DWs are far-enough from each other (the distance is larger than the width of DWs), since then values of 
functions $\left| \pm m_{x}^{(k)} / M-1\right|,\left|m_{y}^{(k)} / M\right|,\left|m_{z}^{(k)} / M\right|$ of the rhs of (7) are much smaller than one.

Let $a \equiv k\left(x_{02}-x_{01}\right)$. Inserting (8) into the density of energy

$$
\begin{aligned}
\mathcal{H} & =\mathcal{H}_{0}+\mathcal{H}_{\mathrm{z}}=\frac{J}{2 M}\left|\frac{\partial \boldsymbol{m}}{\partial x}\right|^{2}+\frac{\beta_{1}}{2 M}\left[M^{2}-(\boldsymbol{m} \cdot \hat{i})^{2}\right] \\
& +\frac{\beta_{2}}{2 M}(\boldsymbol{m} \cdot \hat{j})^{2}-\gamma \boldsymbol{H} \cdot \boldsymbol{m},
\end{aligned}
$$

where $\mathcal{H}_{\mathrm{Z}}$ denotes the Zeeman part of the Hamiltonian, one arrives at the dependence of the energy $E_{0(\mathrm{Z})} \equiv$ $\int_{-\infty}^{\infty} \mathcal{H}_{0(\mathrm{Z})} \mathrm{d} x$ of the initial static state of the DW pair on $a$ (on the distance between the DW centers)

$$
\begin{gathered}
E_{0}(a)=\frac{M\left(\beta_{1}+\theta \beta_{2}\right)}{2 k}\left[I_{1}^{ \pm}(a)+I_{2}^{ \pm}(a)\right] \\
=\frac{M \sqrt{J\left(\beta_{1}+\theta \beta_{2}\right)}}{2} \operatorname{csch}^{2 \mp 1}(a / 2) \\
\times \operatorname{sech}^{2 \pm 1}(a / 2)[-2 a+\sinh (2 a)], \\
E_{\mathrm{Z}}(a)=-\gamma H_{x} \frac{M}{k} I_{3}^{ \pm}(a) \\
=-2 \frac{\gamma H_{x} M \sqrt{J}}{\sqrt{\beta_{1}+\theta \beta_{2}}} a[\operatorname{coth}(a) \operatorname{csch}(a)],
\end{gathered}
$$

where $\theta=1$ for the Néel DWs while $\theta=0$ for the Bloch DWs, and $I_{k}^{ \pm}(a)$ represent integrals written in Appendix. The upper signs correspond to the pair of the DWs of the opposite chiralities, the case $\varphi_{1}=\varphi_{2}$, while the lower signs correspond to the pair of the DWs of opposite chiralities, the case $\varphi_{1}=\varphi_{2} \pm \pi$. According to the plot of energy of the static DW pair (Fig. 1), in absence of the external field, the interaction is attractive when both the DWs are of opposite chiralities since then the energy grows with the distance of separation of the DWs while it is repulsive in the case of like chiralities. Extrema of $E_{0}(a)+E_{\mathrm{Z}}(a)$ correspond to stationary states (vanishing of the interaction of DWs); stable (minima) or unstable ones (maxima).

In both the cases (of opposite and like chiralities), it is possible to create a stationary magnetization bubble applying an external magnetic field in the direction pursuant or opposite to the bubble magnetization, respectively. However, only in the case of $\varphi_{1}=\varphi_{2} \pm \pi$, the function $E(a) \equiv E_{0}(a)+E_{\mathrm{Z}}(a)$ has a minimum at $a \neq 0$ (see Fig. 1). Therefore, only the bubble created by the DWs of like chiralities is stable against external perturbations. This stable bubble corresponds to the final state of the long-term evolution of a breather.

For $\boldsymbol{H} \neq \mathbf{0}$, one can find exact double-Bloch and double-Néel solutions to LLG equation, thus, one can verify our prediction on existence of soft stationary bubbles. Assuming the stationary double-Bloch DW solution and double-Néel DW solution to (3) to be of the form $f=1+v, g=w \mathrm{e}^{k x}+w \mathrm{e}^{-k x}, v=v^{*}$, one finds

$$
|k|=\sqrt{\frac{\beta_{1}+\theta \beta_{2}-\gamma H_{x}}{J}},
$$

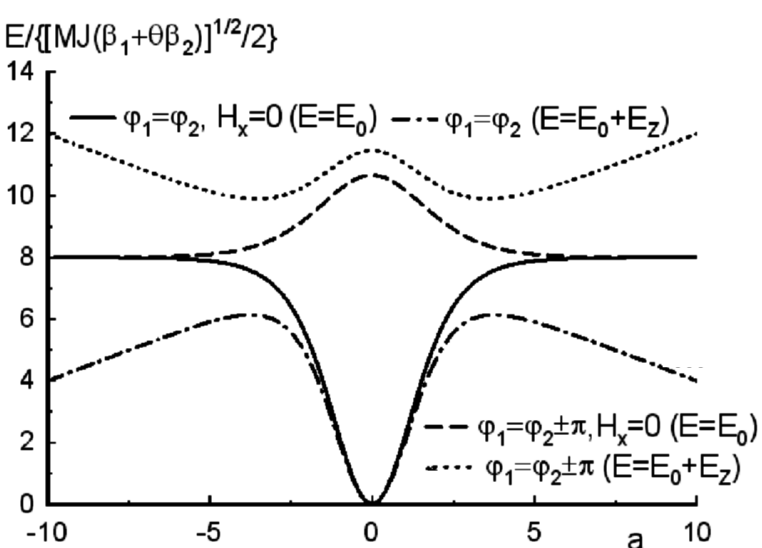

Fig. 1. Energy of a pair of DWs as a function of the distance of their separation; solid (dash-dotted) line DWs of opposite chiralities (an untwisted pair) in absence (presence) of an external field, dashed (dotted) line - DWs of the same chiralities (a twisted pair) in absence (presence) of an external field.

$$
v=-1 \pm 2 \sqrt{\frac{\beta_{1}+\theta \beta_{2}}{\gamma H_{x}}-1}|w|,
$$

for $w=w^{*}, \theta=1$ (an untwisted double-Néel DW), and for $w=-w^{*}, \theta=0$ (an untwisted double-Bloch DW). Inserting $f=1+v, g=w \mathrm{e}^{k x}-w \mathrm{e}^{-k x}$ into (3), one finds

$$
\begin{aligned}
& |k|=\sqrt{\frac{\beta_{1}+\theta \beta_{2}-\gamma H_{x}}{J}}, \\
& v=-1 \pm 2 \sqrt{1-\frac{\beta_{1}+\theta \beta_{2}}{\gamma H_{x}}}|w|,
\end{aligned}
$$

for $w=w^{*}, \theta=1$ (a twisted double-Néel DW), and for $w=-w^{*}, \theta=0$ (a twisted double-Bloch DW). The untwisted double-DWs are called nuclei while the twisted double-DWs are called $2 \pi$-DWs. Since typically $\beta_{1} \gg \beta_{2}$ while $\left|H_{x}\right|=\beta_{1} / \gamma$ corresponds to the coercion-field value (hence, I assume $\left|H_{x}\right| \ll \beta_{1} / \gamma$ ), the untwisted and twisted double-DWs relate to different (parallel or antiparallel to the bubble magnetization, respectively) directions of the external magnetic field, which ensures that $v$ in (12) or (13) is determined. In order that the existence of nuclei was possible, the DWs of like chiralities must attract, thus, the nucleus vanishes after turning the magnetic field off. The prediction on stability of the $2 \pi$-DWs and instability of nuclei is in accordance with a study of linear-excitation scattering on the soft magnetization bubbles and with simulations [18, 20].

In order to complete the picture of the bubble formation in 1D ferromagnet, I refer to my previous study of bound states of one Bloch DW and of one Néel DW [10]. In the absence of external field, such a stationary state is described by a exact solution to the LLG equation, hence, there is no interaction between the walls. An interaction appears, however, upon the field application due to a 


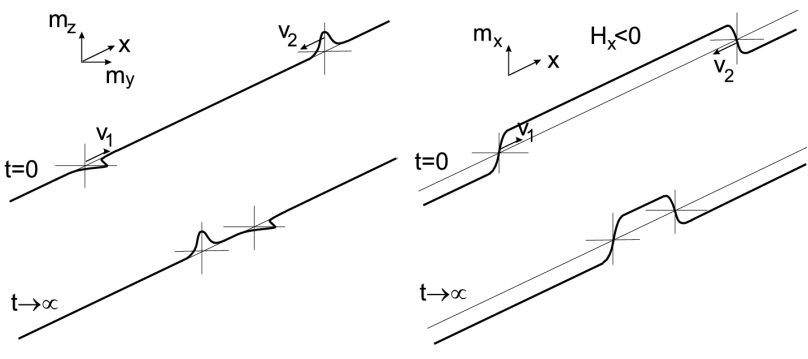

Fig. 2. Formation of hard bubble (composed of one Néel DW and one Bloch DW) in a longitudinal field. The DW reflection is accompanied by a change of the Bloch wall (of the velocity $v_{2}$ ) into the Néel wall (of the velocity $v_{1}$ ) and vice versa.

dynamically induced deformation of the DWs. The external field enforces motion of any DW and its anti-wall in the opposite directions and it can induce the collision of a Néel DW with the Bloch DWs. The result of such a collision has been found to be their mutual reflection accompanied by the transformation of the Bloch wall into the Néel DW and vice versa. The process can be considered as the transmission of the Bloch DW through the Néel DW with change of the head-to-head structure into the tail-to-tail one (and vice versa) which is illustrated in Fig. 2, and it is similar to the collision of spontaneously propagating (in absence of dissipation) DWs [17]. Since, under the action of the external field, the different-type DWs interact repulsively, they can form a bubble which is an $1 \mathrm{D}$ counterpart of the hard bubble of planar magnets.

\section{Bubbles near criticality}

The subcritical dynamics of bistable systems is governed by the GL equation

$$
\alpha \frac{\partial m}{\partial t}=J \frac{\partial^{2} m}{\partial x^{2}}+\beta_{1} m+\beta_{2} m^{*}-\mu|m|^{2} m+\gamma H
$$

Applied to a plane-ordered easy-axis ferromagnet $(m=$ $\left.m_{x}+\mathrm{i} m_{y}\right), J$ denotes an exchange constant, $\beta_{2}$ corresponds to a constant of anisotropy (for the hard direction $y), \beta_{1}+\beta_{2} \sim\left(T_{\mathrm{c}}-T\right) / T_{\mathrm{c}}$ and $\mu$ are the parameters of the Ginzburg-Landau potential that describes a phase transition at a critical temperature $T_{\mathrm{c}}$ (see the energy density below), $\alpha$ denotes a dissipation constant [21, 22]. Also, Eq. (14) is a generic system for a class of the so called "subcritical" wave problems in different branches of physics whose genuine amplitude equations may differ from (14) by adding small imaginary parts to the (real) parameters $\alpha, J, \beta_{1(2)}, \mu[23,24]$.

For $\beta_{1}>3 \beta_{2}$, (14) describes the evolution of the Bloch (of lower energy) and the Ising (of higher energy) DWs and their complexes. The field-induced creation of $1 \mathrm{D}$ hard bubble (composed of one Bloch DW and one Ising DW) has been investigated in [11]. It has been found to be similar to the dynamics of the hard bubble in the low temperature regime. For $H=0$, the stationary state of one Ising DW and one Bloch DW in an 1D subcritical sys- tem is described by an exact solution to (14), thus, both the DWs do not interact [23]. The field-driven collision of the Ising DW with the Bloch DW induces their mutual reflection, hence, they can form a similar hard bubble as it was described in the previous section. I mention that an analysis of linear-wave scattering on the BlochIsing complex with the parametrically-driven nonlinear Schrödinger equation has shown the dynamically-induced repulsion of both the walls, too [25]. Below I pay my attention to soft magnetization bubbles (composed of two Bloch DWs or of two Ising DWs).

In the first perturbation approximation, in the vicinity of the center of $j$-th DW $(j=1,2)$, the magnetization profile can be written as

$$
m(x, 0)=m^{(j)}(x)+\delta m^{(j)}(x),
$$

where $m^{(j)}$ denotes an exact (stationary) single-DW solution to (14) for $H=0$

$$
\begin{aligned}
& m^{(j)}(x)=\sqrt{\frac{\beta_{1}+\beta_{2}}{\mu}} \tanh \left(\sigma_{j} k\left(x-x_{0 j}\right)\right) \\
& \quad+\mathrm{i} \sin \varphi_{j} \sqrt{\frac{\beta_{1}-3 \beta_{2}}{\mu}} \operatorname{sech}\left(\sigma_{j} k\left(x-x_{0 j}\right)\right) .
\end{aligned}
$$

Here $\sigma_{1}=-\sigma_{2}= \pm 1$, and $k=\sqrt{2 \beta_{2} / J}, \varphi_{j}= \pm \pi / 2$ (the Bloch DWs) or $k=\sqrt{\left(\beta_{1}+\beta_{2}\right) /(2 J)}, \varphi_{j}=0, \pi$ (the Ising DWs). According to my claim on the form of the ansatz (8), since the interaction of DWs is related to a reorientation of the separating them domain, I expect the perturbed magnetization not to contain linear in $m^{(j)}$ terms. Taking the following perturbation $\delta m^{(j)}=m^{(j)}\left[ \pm m^{(k)} \sqrt{\mu /\left(\beta_{1}+\beta_{2}\right)}-1\right], k \neq j$, which is small provided the distance between the DWs is larger than their width, leads to

$$
m= \pm \sqrt{\frac{\mu}{\beta_{1}+\beta_{2}}} m^{(1)} m^{(2)} .
$$

This form of the initial magnetization was used in [12], whereas, previous treatments of the DW interactions within the GL approximation used the ansatz $\delta m^{(j)}=$ $m^{(k)} \pm \sqrt{\left(\beta_{1}+\beta_{2}\right) / \mu}, k \neq j$, by an analogy to the perturbation calculus for nontopological (bright) solitons [13].

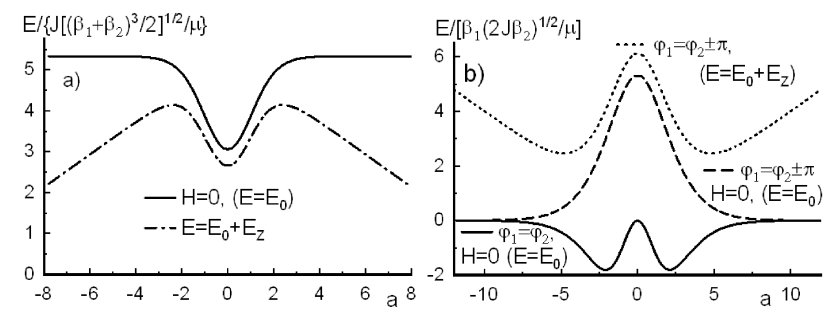

Fig. 3. Energy of a pair of DWs as a function of the distance of their separation: (a) solid (dash-dotted) line - Ising DWs in absence (presence) of an external field, (b) solid line - Bloch DWs of the opposite chiralities (an untwisted pair), dashed (dotted) line - Bloch DWs of the same chiralities (a twisted pair) in absence (presence) of an external field. 
The energy of a temporal static state of the (essentially dissipative, non-Hamiltonian) GL system is defined via the static (independent of the time derivative of $\boldsymbol{m}$ ) part of the Lyapunov functional similar to the energy of the corresponding temporal state of the LLG system. Inserting (17) into the energy density

$$
\begin{aligned}
\mathcal{H} & =\mathcal{H}_{0}+\mathcal{H}_{\mathrm{Z}}=J\left|\frac{\partial m}{\partial x}\right|^{2}+\frac{\mu}{2}\left(|m|^{2}-\frac{\beta_{1}+\beta_{2}}{\mu}\right)^{2} \\
& -\frac{\beta_{2}}{2}\left(m-m^{*}\right)^{2}-\gamma H\left(m+m^{*} \pm 2 \frac{\sqrt{\beta_{1}+\beta_{2}}}{\sqrt{\mu}}\right),
\end{aligned}
$$

one arrives at the dependence of the energy of a static state of two Bloch DWs or of two Ising DWs $E_{0(\mathrm{Z})} \equiv$ $\int_{-\infty}^{\infty} \mathcal{H}_{0(\mathrm{Z})} \mathrm{d} x$ on the distance between the DW centers. Defining $a \equiv k\left(x_{02}-x_{01}\right)$, for the interacting Ising DWs, one finds

$$
\begin{aligned}
& E_{0}(a)=\frac{\left(\beta_{1}+\beta_{2}\right)^{2}}{2 \mu k} I_{4}(a) \\
& =\frac{\sqrt{J}\left(\beta_{1}+\beta_{2}\right)^{3 / 2}}{3 \sqrt{2} \mu} \operatorname{coth}^{2}(a) \operatorname{csch}^{5}(a)[120 a \cosh (a) \\
& \quad-80 \sinh (a)-15 \sinh (3 a)+\sinh (5 a)] \\
& E_{\mathrm{Z}}(a)=-2 \gamma H \frac{\sqrt{\beta_{1}+\beta_{2}}}{\sqrt{\mu} k} I_{5}(a) \\
& =-4 \gamma H \frac{\sqrt{2 J}}{\sqrt{\mu}} a \operatorname{coth}(a)
\end{aligned}
$$

while for the Bloch DWs ( $E_{0}$ is derived up to the lowest order in $\left.\beta_{2} / \beta_{1}\right)$;

$$
\begin{aligned}
& E_{0}(a)=\frac{2 \beta_{1} \beta_{2}}{\mu k}\left[I_{1}^{ \pm}(a)-I_{2}^{ \pm}(a)\right] \\
& =\frac{\beta_{1} \sqrt{2 J \beta_{2}}}{\mu} 2 \operatorname{csch}^{2 \mp 1}(a / 2) \\
& \quad \times \operatorname{sech}^{2 \pm 1}(a / 2)[-a \cosh (a)+\sinh (a)], \\
& E_{\mathrm{Z}}(a)=-2 \gamma H \frac{\sqrt{\beta_{1}+\beta_{2}}}{\sqrt{\mu} k} I_{3}^{ \pm}(a) \\
& \quad=-4 \gamma H \frac{\sqrt{J\left(\beta_{1}+\beta_{2}\right)}}{\sqrt{2 \beta_{2} \mu}} a[\operatorname{coth}(a) \mp \operatorname{csch}(a)] .
\end{aligned}
$$

The integrals $I_{k}(a)$ are written in Appendix. Here, the upper signs correspond to a pair of the Bloch walls of opposite chiralities, the case $\varphi_{1}=\varphi_{2}$ while the lower signs to a pair of the Bloch walls of like chiralities, the case $\varphi_{1}=\varphi_{2} \pm \pi$. Plotting the energy $E_{0}(a)$ of the DW pairs (Fig. 3), we see the Ising DWs to attract each other in the absence of external field while the character of the interaction of the Bloch DWs to be dependent on their chiralities. In the presence of an external field parallel to the bubble magnetization, the Ising walls can form a stationary state corresponding to the maximum of energy, however, it is unstable to perturbations. The Bloch DWs of opposite chiralities attract each other when the distance of their separation is big and they repel each other at short separation distances. Because of the minima of the energy $E_{0}(a)$ at $a \neq 0$, stable bubble of the Bloch DWs can be formed even in absence of the field. The Bloch DWs of like chiralities always repel in absence of the field, hence, an external field antiparallel to the magnetization of the intermediate domain enables creation of a stable bubble which corresponds to the minima of $E_{0}(a)+E_{\mathrm{Z}}(a)$.

\section{Structures of many DWs}

Stability of many-DW structures is an important problem in view of the challenge of designing a nanowire-based information carrier (a DW-racetrack memory) [3]. Periodic structures of the Bloch (Néel) DWs are stationary because of the compensation of (attractive or repulsive) interactions between the walls. However, bit recording requires switching the magnetization of a single domain of the memory tape on demand. For instance, the magnetization reversal can be performed via flip of the chirality of two neighboring DWs in the tape, which initiates their movement towards or outwards each other and, eventually, results in their annihilation due to the appearance of uncompensated attractive forces. Unfortunately, the resulting nonperiodicity of the system causes destabilization of the record. I mention that similar process is responsible for annihilation of DWs during strong-current driven motion of multidomain systems [26]. Then, the unbalanced interactions appear because, for the current intensity exceeding the Walker-breakdown value, magnetization in different (head-to-head and tail-to-tail) DWs rotates in opposite directions about the magnetic axis (unlike upon the application of a strong magnetic field).

An exception is a subcritical system of the Bloch DWs whose all the neighboring DWs are of opposite chiralities and the distance of their separation corresponds to the size of the breather found in Sect. 3. The result of changing the chirality of a single DW or a DW pair is some shift of the wall positions without any flip of the domains.

Aperiodic trains of DWs of like chiralities (unstable to DW interactions) as well as trains of alternating Bloch and Néel (Ising) DWs (unstable to the Néel-Bloch or Ising-Bloch transition inside the DWs [22]) can be stabilized by an external magnetic field whose application results in creation of soft and hard magnetization bubbles, respectively. The energy of a soft bubble increases with the field value (see minima of $E(a)$, dotted lines in Fig. 1 and Fig. 3), hence, unlike for weak fields, in a regime of strong field, the hard bubbles can be energetically favorable [8].

\section{Acknowledgments}

This work was supported by Polish Government Research Funds for 2010-2012 in the framework of grant No. N N202 198039. 


\section{Appendix A: Explicit form of integrals}

$$
\begin{aligned}
& I_{1}^{ \pm}(a) \equiv \int_{-\infty}^{\infty}\left(\left\{\operatorname{sech}^{2}(y) \tanh (-y+a)\right.\right. \\
& \quad-\operatorname{sech}^{2}(-y+a) \tanh (y) \mp[-\tanh (y) \\
& +\tanh (-y+a)] \operatorname{sech}(y) \operatorname{sech}(-y+a)\}^{2} \\
& \quad+\{-\operatorname{sech}(y)[\tanh (y) \tanh (-y+a) \\
& \left.\quad+\operatorname{sech}^{2}(-y+a)\right] \\
& \left.\quad \pm \operatorname{sech}^{-y}+a\right)[\tanh (y) \tanh (-y+a) \\
& \left.\left.\left.\quad+\operatorname{sech}^{2}(y)\right]\right\}^{2}\right) \mathrm{d} y, \\
& I_{2}^{ \pm}(a) \equiv \int_{-\infty}^{\infty}([\operatorname{sech}(y) \tanh (-y+a) \\
& \left.\left.\quad \pm \tanh ^{\infty}(y) \operatorname{sech}(-y+a)\right]^{2}\right) \mathrm{d} y, \\
& \left.\quad+\left[\tanh ^{2}(y) \tanh (-y+a)-1\right]^{2}\right\} \mathrm{d} y, \\
& I_{3}^{ \pm}(a) \equiv \int_{-\infty}^{\infty}[\tanh (y) \tanh (-y+a) \\
& \left.\quad \mp \operatorname{sech}^{2}(y) \operatorname{sech}(-y+a)+1\right] \mathrm{d} y, \\
& I_{5}(a) \equiv \int_{-\infty}^{\infty}[\tanh (y) \tanh (-y+a)+1] \mathrm{d} y . \operatorname{sech} \int_{-\infty}^{2}\{[\operatorname{sech}(y) \tanh (-y+a)
\end{aligned}
$$

\section{References}

[1] P. Coullet, C. Riera, C. Tresser, Chaos 14, 193 (2004).

[2] A.S. Mikhailov, K. Showalter, Phys. Rep. 425, 79 (2006).

[3] S.S.P. Parkin, M. Hayashi, L. Thomas, Science 320, 190 (2008).

[4] M. Hayashi, L. Thomas, R. Moriya, C. Rettner, S.S.P. Parkin, Science 320, 209 (2008).

[5] J.F. Scott, Science 315, 954 (2007); A. Schilling, R.M. Bowman, G. Catalan, J.F. Scott, J.M. Gregg, Nano Lett. 7, 3787 (2007).

[6] A. Thiaville, J.M. Garcia, J. Miltat, J. Magn. Magn. Mater. 242-245, 1061 (2002); A. Thiaville, Y. Nakatani, J. Miltat, Y. Suzuki, Europhys. Lett. 69, 990 (2005).
[7] R.G.P. McQuaid, M. McMillen, L.-W. Chang, A. Gruverman, J.M. Gregg, J. Phys., Condens. Matter 24, 024204 (2012).

[8] A. Rosencwaig, W.J. Tabor, T.J. Nelson, Phys. Rev. Lett. 29, 946 (1972).

[9] F.H. de Leeuw, R. van der Doel, U. Enz, Rep. Prog. Phys. 43, 689 (1980).

[10] A. Janutka, Phys. Rev. E 83, 056607 (2011).

[11] A. Janutka, Phys. Rev. E 83, 056608 (2011).

[12] I.V. Barashenkov, S.R. Woodford, E.V. Zemlyanaya, Phys. Rev. E 75, 026604 (2007).

[13] L. Korzinov, M.I. Rabinovich, L.S. Tsimring, Phys. Rev. A 46, 7601 (1992); H. Tutu, H. Fujisaka, Phys. Rev. B 50, 9274 (1994); B.A. Malomed, A.A. Nepomnyashchy, Europhys. Lett. 27, 649 (1994).

[14] A. Janutka, Domain-Wall Complexes in Ferromagnetic Stripes, arXiv:1201.5760.

[15] M.M. Bogdan, A.S. Kovalev, JETP Lett. 31, 424 (1980); R. Hirota, J. Phys. Soc. Jpn. 51, 323 (1982).

[16] T.A. Moore, I.M. Miron, G. Gaudin, G. Serret, S. Auffret, B. Rodmacq, A. Schuhl, S. Pizzini, J. Vogel, M. Bonfim, Appl. Phys. Lett. 93, 262504 (2008); L. Thomas, S.-H. Yang, K.-S. Ryu, B. Hughes, C. Rettner, D.-S. Wang, C.-H. Tsai, K.-H. Shen, S.S.P. Parkin, in: Proc. IEEE International Electron Devices Meeting IEDM 2011, Washington 2011, p. 24.2 .1 .

[17] A.M. Kosevich, B.A. Ivanov, A.S. Kovalev, Phys. Rep. 194, 117 (1990); M. Svendsen, H. Fogedby, J. Phys. A 26, 1717 (1993).

[18] H.-B. Braun, Phys. Rev. B 50, 16485 (1994).

[19] J.L. Eves, N. Grisewood, R. Hugli, H.-B. Braun, J. Magn. Magn. Mater. 322, 1381 (2010).

[20] M.A. Shamsutdinov, V.N. Nazarov, I.Yu. Lomakina, Phys. Met. Metallogr. 101, 309 (2006); M.A. Shamsutdinov, V.N. Nazarov, ibid., 107, 569 (2009).

[21] L.N. Bulaevskii, V.L. Ginzburg, Sov. Phys. JETP 18, 530 (1964).

[22] J. Lajzerowicz, J.J. Niez, J. Phys. Lett. 40, L165 (1979).

[23] I.V. Barashenkov, S.R. Woodford, Phys. Rev. E 71, 026613 (2005).

[24] M. Ipsen, L. Kramer, P.G. Sorensen, Phys. Rep. 337, 193 (2000).

[25] I.V. Barashenkov, S.R. Woodford, Phys. Rev. E 75, 026605 (2007).

[26] H. Murakami, K. Takahashi, T. Komine, R. Sugita, J. Phys., Conf. Ser. 200, 042018 (2010); M.D. Mascaro, C.A. Ross, Phys. Rev. B 82, 214411 (2010). 